\title{
Effect of non-immunogenic microenvironmental factors on tumor growth dynamics modeled by correlated noises in the presence of immune response
}

\begin{abstract}
The steady state properties for the effect of non-immunogenic microenvironmental factors on tumor growth dynamics in the presence of immune response is investigated. The corresponding Fokker-Planck equation to the Langevin model equation interpreted in the sense of Stratonovich is used to derive the steady state distribution $f \mathrm{st}(\mathrm{x})$ and the mean $\langle\mathrm{x}$ $>$ st of the tumor growth system. We find that the correlation strength ${ }^{U}$ stimulates the effect of the non-immunogenic microenvironmental factors ů on the tumor growth dynamics, and the tumor response $\mathrm{M}$ to the non-immunogenic microenvironmental factors within the tumor site may inhibits tumor growth, but not sufficient enough to cause extinction. Moreover, the result also indicates that the stronger the immune response $z$ the more the tumor population disappears.
\end{abstract}

Keyword: Tumor growth dynamics; Immune response; Non-immunogenic microenvironmental factors 